\title{
A Novel Technique to Enhance the Lifetime of Wireless Sensor Networks through Software Realization
}

\author{
Adarsh Sagar' ${ }^{1}$ T. G. Basavaraju ${ }^{2}$, K. B. Surekha ${ }^{3}$ \\ ${ }^{1}$ Department of CSE, APSCE, Bangalore, India \\ ${ }^{2}$ Department of CSE, GSKSJTI, Bangalore, India \\ ${ }^{3}$ Department of CSE, Acharya IT, Bangalore, India
}

\begin{tabular}{l} 
Article Info \\
\hline Article history: \\
Received Feb 15, 2018 \\
Revised May 20, 2018 \\
Accepted May 28, 2018 \\
\hline
\end{tabular}

Keyword:

Aggregation

Coverage

Software realization

WSN

\begin{abstract}
In the most of the real world scenarios, wireless sensor networks are used. Some of the major tasks of these types of networks is to sense some information and sending it to monitoring system or tracking some activity etc. In such applications, the sensor nodes are deployed in large area and in considerably large numbers [1]-[3]. Each of these node will be having constrained resources whether it might be energy, memory or processing capability. Energy is the major resource constraint in these types of networks. Hence enough care to be taken in all aspects such that energy can be used very efficiently. Different Activities which will be taking place in a sensor node are sensing, radio operations and receiving and computing. Among all these operations, radio consumes maximum power. Hence there is a need of reducing the power consumption in such radio operations. In the proposed work a software module is developed which will reduce the number of transmissions done to the base station. The work compares the consecutively sensed data and if these data are same then the old data then the old data will be retained. In other case the newly sensed data will be sent to the sink node. This technique reduces the number of data transmissions in a significant way. With the reduced number of transmissions, the energy saved in each node will be more, which will increase the lifetime of the entire network.
\end{abstract}

Copyright $@ 2018$ Institute of Advanced Engineering and Science. All rights reserved.

\section{Corresponding Author:}

K. B. Surekha,

Department of CSE,

Acharya IT,

Bangalore, India.

Email: surekha@acharya.ac.in

\section{INTRODUCTION}

Wireless Sensor Network consists of small nodes called as sensors (motes) which are used for varieties of applications. Some of the applications are monitoring a particular part of the area, objects, people, sensing the humidity, temperature. The deployment of these sensor nodes in the required application will be in large numbers. The reason for large quantity of deployment is to maintain the coverage of the network. Even physical theft of nodes and also the energy will drain of the sensor node at a faster makes the deployment of the nodes in a larger extent.

In sensor networks, energy is one of the most precious resources and it's a critical issue also. Hence in this proposed work an attempt is been made to reduce the usage of energy by every node. The major activities which are going to take place in every sensor node is sensing, transmitting, receiving, idle and sleep. Out of all these activities, transmission consumes most of the energy. So, in this proposed work a software module is developed, where the number of transmissions to the base station are reduced which will save the energy of the node. Our objective in this work is to minimize the usage of the energy and increase the network lifetime. 


\section{EXISTING WORK}

Amrita Ruperee et al. [4] have discussed about the comparator which reduces the length of the data before transmitting it to the base station. The proposed method compares the previously sensed data with the present data using delta modulator. The proposed technique is applicable to hierarchical network, where clusters are defined.

Kiran Mariaya [5] have discussed about the issues in data aggregation process. The author has also listed the mean to measure the performance of data aggregation, how data aggregation impacts on WSN. Different data aggregation function used in WSN are (a) duplicate sensitive and duplicate insensitive, which used several duplication functions to remove redundancy (b) lossy and lossless approach where compression of data collected will be done at the nodes. The procs and cons of the data aggregation process is also listed in the work.

Krishnamachari et al. [6] derive a model for data centric routing which is different from normal address centric routing. In address centric routing, packets will be routed from one hop to other based on the destination address. The routing path will be searched based on the destination address and the packet will be reaching to the destination. Where as in data centric routing, routing nodes, check the content of the packet. If the packet are from same source along the same route to the destination, aggregation will be done in the routing process it self. This process will eliminate the redundant data. The author has also given the performance measure for the entire data aggregation process. They are the amount of energy saved due to entire process of data transmission, and the latency encountered in the data aggregation process.

Akimtsu et al. [7] have proposed a new way of reducing the number of data transmission. This technique is called as ODAS (Overhearing based data aggregation method using spatial interpolation). The theme work well for wireless networks, because, the data transmission in wireless networks happens usually in omni directions. Or in other words, if one node is in the transmission process, the neighboring nodes overhear this transmission. The method identifies the spatially correlated nodes. The sensed value of the actual transmitted node and the reading of the overhearing node will be compare. If the resulting data is within acceptable range, the data will be transmitted only once. This will reduce the number of data transmission to the greater extent.

Similarity based Adaptive Framework [8] technique works based on time series forecasting model. The technique tries to reduce the number of data transmission based on the predicting the sensor readings. The predicted or forcasted readings might prove wrong in some cases. But in some applications like, temperature sensing, during a particular season, it can be predicted and in most of the cases it may be prove correct also. Again the technique will reduce the transmission of data to the sink, which reduces the usage energy.

\section{PROPOSED WORK}

The availability of energy is the most critical issue, which makes us think the usage of energy in more efficient manner. Experimental results have shown that among all the operations in sensor network like sensing, transmitting and receiving, transmitting consumes more energy compared to all the operations.

In the work [4], [9], a comparator is designed which will compare the previously sensed data with the present data. If both data are same or within acceptable range then a handshaking signal is sent, by just sending a bit 0 . Otherwise the sensed data will be sent. This will reduce the number of data transmissions and increases the lifetime of the sensor node.

In the work [9], the hardware is proposed to compare the subsequent sensed data and decision is made to transmit the data or not. The hardware incorporated for comparing the data consumes additional energy of $0.088 \mu$ Joules for each comparison. The benefit of the method is demonstrated using the temperature sensing application.

The proposed idea is brought to realization using the microprocessor instruction set. By considering the MSP430 micro controller [10] is the idea is arrived at the implementation. The Figure 1 shows the routine gives the set of for the same.

$$
\begin{aligned}
& \text { above: Cmp.b reg4,[port b] } \\
& \text { Jeq Neext } \\
& \text { Mov.b[port b],reg4 } \\
& \text { Neext: jmp above }
\end{aligned}
$$

Figure 1. Set of instruction for the proposed work 
The actions of the above micro controller instructions set is been explained below. The newly sensed data will be present any port of the micro control. Let us say the information is saved in port B. the propose method is made to save the previously sensed value in the any one general purpose register of the the micro controller.

Now the newly sensed data which is made to sit in port B is compared with the previous data which is stored in register. If the compared values are same or within the tolerable limit, then the present data which is available in port will not be saved or neither be transmitted. This act will tell that there is no need of data transmission in this case. In the other case, i.e if the difference value of sensed data is beyond the tolerable limit, the presently sensed data will be transmitted to the sink. Apart from this transmission operation, the microcontroller has to save a copy of this for further comparison in the next iteration. This process will reduce the number of data transmissions to the grater extent.

Now let us analyze the energy consumption for the proposed technique. Usually, to fetch a single instruction from the EEPROM, the amount of energy consumed will be $0.10509 \mu$ Joules [11]. The voltage required to pull the data will be ranging from $3 \mathrm{~V}$ to $3.3 \mathrm{~V}$. The current drawn for each such instruction will be $6.2 \mathrm{~mA}$. The time consumed to draw on instruction from EEPROM will be is $565 \mu$ s [11]. Hence the entire process of fetching the instruction and executing in the main memory will consume $\mathrm{v} * \mathrm{i} * \mathrm{t}$ amount of energy. By substituting the standard values, the energy consumption calculated will arrive at $0.1059 \mu$ Joules. The proposed technique involves 4 instructions. Hence the total energy consumed by the entire routine will reach at $0.42 \mu$ Joules.

Table 1. Comparison with the Hardware Module

\begin{tabular}{ccc}
\hline Data recorded for the year(No of times) & $\begin{array}{c}\text { Energy dissipation with the hardware } \\
\text { module[9] (in } \mu \text { Joules) }\end{array}$ & $\begin{array}{c}\text { Energy dissipation with the software } \\
\text { routine (in } \mu \text { Joules) }\end{array}$ \\
\hline $2011(18)$ & 1.584 & 7.56 \\
$2012(16)$ & 1.408 & 6.72 \\
$2013(10)$ & 0.88 & 4.2 \\
$2014(14)$ & 1.232 & 5.88 \\
$2015(15)$ & 11.25 & 15.98 \\
\hline
\end{tabular}

Table 1 gives the comparison of energy savings with hardware module [9] and the software routine. The data collected is for temperature sensing application [9]. The data collected is for the day of January Ist in different years like 2011, 2012 .. 2015, how many times data was sent (shown in brackets). For example if we take for the year 2011, data sent was only 18 times (against normal 24 times). In case of the hardware module [9] the energy dissipation was $1.584 \mu \mathrm{J}$ oules, where as in case of software routine the energy dissipation is $7.56 \mu$ Joules. The overhead incurred in software method is almost 5 times higher than the hardware design.

\section{CONCLUSION}

Reducing the number of data transmissions is always better in WSN, as the data transmission consumes more energy when compared to all the other operations in the sensor networks. This also will reduce the usage of bandwidth also, as bandwidth is also one of the constrain resource. In this work the hardware circuit [9] is compared with the software realization of the same. It is been proved that software implementation consumes considerably more energy when it is compared with the hardware design.

\section{REFERENCES}

[1] I. Akyildiz, et al., "A Survey on Sensor Networks", Communications Magazine, IEEE, vol. 40, no. 8, pp. 102-114, 2002.

[2] Al-Karaki, et al., "Routing Techniques in Wireless Sensor Networks: A Survey", IEEE Wireless Communications, vol. 11, no. 6, pp. 6-28, 2004.

[3] Guo-Xing Liu and Zhen-Wei Yu, "Survey on Wireless Sensor Networks", IEEE, International Conference on Internet Technology and Applications, 2011.

[4] Amrita Ruperee, et al., "Achieving Energy Efficiencyand Increasing Network Life in Wireless Sensor Network", IEEE International Advance Computing Conference (IACC), 2014.

[5] Kiran Maraiya, et al., "Wireless Sensor Network: A Review on Data Aggregation", International Journal of Scientific \& Engineering Research, vol. 2, no. 4, April 2011, ISSN-2229-5518.

[6] Krishnamachari, L., et al., "The Impact of Data Aggregation in Wireless Sensor Networks", Distributed Computing Systems Workshops, Proceedings 22nd International Conference on. IEEE, 2002. 
[7] Kanzaki, et al. "Overhearing-based Data Transmission Reduction using Data Interpolation in Wireless Sensor Networks", The 5th Int'l conference on Mobile Computing and Ubiquitious Networking, vol. 2010, 2010.

[8] Gennaro Boggia, et al., "Power Efficiency of the Similarity-based Adaptive Framework in Wireless Sensor Networks", Proc. of Wireless Rural and Emergency Communications, WRECOM 2007, Rome, Italy, Oct., 2007.

[9] Surekha K. B., et al., "Minimization of Transmission Energy by exploiting Redundancy in Temporal Data", ICDECS-2015, International Journal of Applied Engineering Research, vol. 10, no. 56, 2015, ISSN 0973-4562.

[10] Davies, John H., "MSP430 microcontroller basics” Elsevier, 2008.

[11] Nicholas D. Lane and Andrew T. Campbell, "The Influence of Microprocessor Instructions on the Energy Consumption of Wireless Sensor Networks". 